\title{
ANALYTICAL EXPRESSIONS FOR EXCITATION AND IONIZATION CROSS-SECTIONS AND RATE COEFFICIENTS OF HYDROGEN-LIKE IONS BY ELECTRON AND PROTON IMPACT
}

\author{
A. JACOBS* \\ Space Research Laboratory, Utrecht, The Netherlands
}

(Received 16 October 1970)

\begin{abstract}
Excitation and ionization cross-sections in the Bethe-Born approximation have been computed for a model of the hydrogen-like ion consisting of seven discrete energy levels and a continuum.

We propose an interpolation formula connecting the high energy behaviour (Bethe-Born) of the cross-sections and their values at threshold (Coulomb-Born I). We compare this formula with other-frequently used-formulae. Excitation and ionization rate coefficients in a Maxwellian plasma have been computed.
\end{abstract}

\section{INTRODUCTION}

ExcITATION cross-sections by electron impact for some transitions from the ground state of a large number of astrophysically important positive ions have already been calculated (e.g. Bely, ${ }^{(2)}$ Bely and BeLY, ${ }^{(3)}$ BeLY and BlaHA, ${ }^{(4)}$ and the review by Morserwitsch and SMITH. ${ }^{(21)}$ For ionization see the review by RUDGE ${ }^{(23)}$ ).

However, relatively little theoretical work has been published on cross-sections of hydrogen-like ions. TuLl $Y^{(25)}$ calculated the $1 s-2 s$ cross-section, Burgess ${ }^{(6)}$ the $1 s-2 p$ cross-section. BuRKE et al. computed the excitation cross-sections in the close-coupling approximation for the array $1 s-2 s-2 p-3 s-3 p-3 d$ of $\mathrm{He}^{+}$. The cross-section for ionization from the ground state in various approximations has been investigated by BuRGESs and RUDGE. ${ }^{(8)}$

In the last few years a large number of solar spectra have been obtained in the extreme u.v. region by means of rocket and satellite borne spectrographs. In these spectra lines belonging to the Lyman series of C VI, N VII, O VIII, Ne X and Fe XXVI have been identified. For the correct interpretation of the intensities of these lines reliable excitation and ionization rate coefficients are indispensable. Until now SEATON's ${ }^{(24)}$ excitation rate formula has been used most frequently. This formula is based on a false picture of the behaviour of the excitation cross-section at threshold as will be shown in Section 9. The most widely used formula for the ionization rate is that of BuRGess and SEATON, ${ }^{(9)}$ though a better formula-with a more accurate $Z$-dependence-(BuRGEss et al. ${ }^{(7)}$ ) has appeared occasionally in the literature. In Section 9 it will be shown that these two formulae can be

\footnotetext{
* Present address: Department of Environmental Sciences, University of Tel Aviv, Ramat Aviv, Israel.
} 
made to give the exact low temperature behaviour of the ionization rate by adapting the numerical factor and that their $n$ (level)-dependence, although oversimplified, is quite accurate.

In the next three sections we shall describe how to compute the two parameters of the Bethe-Born formula for excitation according to BeTHE ${ }^{(5)}$ and MILLER and PlATzMAN, ${ }^{(20)}$ for the total inelastic cross-section according to INOKUTI et al. ${ }^{(15)}$ and for the ionization cross-section. In Section 5 we shall propose an interpolation formula connecting the highenergy formula with the value of the cross-section at threshold. In Section 6 we shall derive formulae for the excitation and ionization cross-sections by proton impact, in Section 7 we shall derive the expressions for the rate coefficients, in Section 8 we shall treat the $2 s-2 p$ transitions and in Section 9 we shall compare our results with other well-known expressions from literature.

We shall use atomic units unless specified otherwise. Cross-sections however will be expressed as customary in units $Z^{-4} \pi a_{0}^{2}$, except in the first part of the next section.

We shall neglect cxchange contributions to the Bethe-Born cross-sections becausc they are of higher nullity with respect to energy than the retained direct terms.

\section{HIGH ENERGY EXCITATION CROSS-SECTIONS BY ELECTRON IMPACT}

The Born I cross-section for excitation from the term $n l-n^{\prime} l^{\prime}$ is

$$
\sigma_{\mathrm{ex}}^{n l, n^{\prime} l^{\prime}}(E)=\frac{4 \pi}{E} \int_{k_{-}}^{k_{+}} \frac{F_{n l, n^{\prime} l^{\prime}}^{2}(k)}{k^{3}} \mathrm{~d} k
$$

$E$ is the kinetic energy of the incident electron.

$$
\begin{aligned}
k_{ \pm}= & \sqrt{ }(2 E) \pm \sqrt{ }\left[2\left(E-E_{t}\right)\right] . \\
E_{t}= & \frac{1}{2} Z^{2}\left(n^{-2}-n^{\prime-2}\right), \text { the transition or threshold energy. } \\
& F_{n l, n^{\prime} l^{\prime}}^{2}(k)=(2 l+1)^{-1} \sum_{m=-1<}^{+1<}\left|\left\langle n l m\left|\mathrm{e}^{i \mathbf{k} \cdot r}\right| n^{\prime} l^{\prime} m\right\rangle\right|^{2} .
\end{aligned}
$$

We have chosen the $z$-axis parallel to $\mathbf{k}$, so that the matrix of $\mathrm{e}^{\mathbf{i k . r}}$ is diagonal in $m . l_{<}$is the smaller of $l$ and $l^{\prime}$.

In order not to make the formulae unnecessarily obscure we shall omit the indices referring to levels $(n)$, terms $(n l)$ or states $(n l m)$. For hydrogen-like ions with charge $Z-1$, $F^{2}(k)$ contains $k$ and $Z$ only in the combination $(k / Z)^{2}$. We define

$$
\begin{gathered}
t^{-2}=n^{-2}-n^{\prime-2}, \\
\alpha=1 / n+1 / n^{\prime}, \\
U=E / E_{t},
\end{gathered}
$$


and substitute into (1)

$$
\begin{aligned}
x & =k^{2} /\left(\alpha^{2} Z^{2}\right) \\
\sigma_{\mathrm{ex}}(U) & =\frac{4 \pi t^{2}}{Z^{4} U \alpha^{2}} \int_{x_{-}}^{x_{+}} \frac{F^{2}(x)}{x^{2}} \mathrm{~d} x \\
x_{ \pm} & =\frac{2}{t^{2} \alpha^{2}}\left\{U-\frac{1}{2} \pm \sqrt{ }[U(U-1)]\right\}
\end{aligned}
$$

From now on we shall express $\sigma$ in units $Z^{-4} \pi a_{0}^{2}$ and therefore drop the factor $\pi Z^{-4}$ in (5). $\left(a_{0}^{2}\right.$ is the atomic unit of area, the unit in which (5) is expressed and therefore does not appear in the formula.) The function $F^{2}(x) / x(\alpha t)^{2}$ is usually called the generalized oscillator strength. $F^{2}(x)$ is a rational function of $x$ :

$$
\begin{gathered}
F^{2}(x)=(x+1)^{-2\left(n+n^{\prime}\right)} \sum_{p=p_{-}}^{p_{+}} a_{p} x^{p} . \\
p_{-}=\left|l-l^{\prime}\right| \text {. If } l=l^{\prime} \text { and } n \neq n^{\prime} \text { then } p_{-}=2 . \\
p_{+}=2\left(n+n^{\prime}-2\right)-l-l^{\prime} .
\end{gathered}
$$

The coefficients $a_{p}$ are rational numbers for which an algorithm will be given in the Appendix.

In the Born-Bethe approximation the limiting forms of (5) and (6) are taken for $U \rightarrow \infty$ :

$$
\begin{aligned}
& x_{-} \rightarrow\left(4 t^{2} \alpha^{2} U\right)^{-1} \\
& x_{+} \rightarrow \infty .
\end{aligned}
$$

Performing the integration in (5) one obtains

$$
\sigma_{\mathrm{ex}}=\frac{4 t^{2}}{U \alpha^{2}}\left[\sum_{n=2}^{p_{+}} \frac{a_{p}(p-2) !\left(2 n+2 n^{\prime}-p^{\prime}\right) !}{\left(2 n+2 n^{\prime}-1\right) !}-a_{1} \sum_{k=1}^{2 n+2 n^{\prime}-1} \frac{1}{k}+a_{1} \ln \left(4 t^{2} \alpha^{2} U\right)\right] .
$$

We write this expression concisely

$$
\sigma_{\mathrm{ex}}(U)=\frac{1}{U}(a+b \ln (U))
$$

Or, equivalently, as function of the kinetic energy $E$ :

$$
\sigma_{\mathrm{ex}}(E)=\frac{2}{E}\left(M_{\mathrm{ex}}^{2} \ln c_{\mathrm{ex}}+M_{\mathrm{ex}}^{2} \ln (8 E)\right)
$$

where

$$
\begin{aligned}
M_{\mathrm{ex}}^{2} & =\frac{1}{2} E_{t} b \\
\ln c_{\mathrm{ex}} & =a / b-\ln \left(8 E_{t}\right) .
\end{aligned}
$$

$b$ is simply related to the dipole oscillator strength $f(0)$ :

$$
b=4 t^{4} f(0)
$$


For most application one needs mean cross-sections for transitions between the levels $n$ and $n^{\prime}$. Mean values of $a$ and $b$ are obtained by summing over all values of $l^{\prime}$ and averaging over $l$. The terms $2 s$ and $2 p$ should however be considered separately in plasma-astrophysical applications because of the (optical) metastability of $2 s$.

Values of $a$ and $b$ can be found in Table 1 .

TABLE 1. $a, b, c$ AND $p$ FOR EXCITATION

\begin{tabular}{|c|c|c|c|c|}
\hline Transition & $a$ & $b$ & $c$ & $p$ \\
\hline $1 s-(2 s+2 p)$ & $0.118851^{+1}$ & $0.295962^{+1}$ & $0.287246^{+1}$ & $1 \cdot 140$ \\
\hline $1 s-2 p$ & $0.596581^{+0}$ & $0.295962^{+1}$ & $0.229420^{+1}$ & $1 \cdot 113$ \\
\hline $1-3$ & $0.398893^{+0}$ & $0.400452^{+0}$ & $0.510774^{+0}$ & 0.843 \\
\hline $1-4$ & $0.159171^{+0}$ & $0.131941^{+0}$ & $0.183504^{+0}$ & 0.707 \\
\hline $1-5$ & $0.789029^{-1}$ & $0.604963^{-1}$ & $0.874579^{-1}$ & 0.623 \\
\hline $1-6$ & $0.448089^{-1}$ & $0.330062^{-1}$ & $0.487136^{-1}$ & 0.528 \\
\hline $1-7$ & $0.278885^{-1}$ & $0.200665^{-1}$ & $0.299743^{-1}$ & 0.475 \\
\hline $2 s-3$ & $0.983929^{+2}$ & $0.901737^{+2}$ & $0.148968^{+3}$ & 1.125 \\
\hline $2 s-4$ & $0.194536^{+2}$ & $0.116923^{+2}$ & $0.252616^{+2}$ & 1.708 \\
\hline $2 s-5$ & $0.743269^{+1}$ & $0.380318^{+1}$ & $0.914639^{+1}$ & $2 \cdot 124$ \\
\hline $2 s-6$ & $0.370862^{+1}$ & $0.175197^{+1}$ & $0.445032^{+1}$ & 7.297 \\
\hline $2 s-7$ & $0.214258^{+1}$ & $0.966784^{+0}$ & $0.253556^{+1}$ & 35.01 \\
\hline $2 p-3$ & $0.533919^{+2}$ & $0.147096^{+3}$ & $0.190546^{+3}$ & 1.287 \\
\hline $2 p-4$ & $0.259591^{+2}$ & $0.142040^{+2}$ & $0.309684^{+2}$ & 0.537 \\
\hline $2 p-5$ & $0.105943^{+2}$ & $0.413458^{+1}$ & $0.110470^{+2}$ & 0.372 \\
\hline $2 p-6$ & $0.536905^{+1}$ & $0.180203^{+1}$ & $0.533740^{+1}$ & 0.30 \\
\hline $2 p-7$ & $0.311818^{+1}$ & $0.963166^{+0}$ & $0.303879^{+1}$ & 0.28 \\
\hline $3-4$ & $0.579976^{+3}$ & $0.142545^{+4}$ & $0.217584^{+4}$ & 1.43 \\
\hline $3-5$ & $0.263961^{+3}$ & $0.119114^{+3}$ & $0.326314^{+3}$ & 0.86 \\
\hline $3-6$ & $0.104614^{+3}$ & $0.321640^{+2}$ & $0.113291^{+3}$ & 0.54 \\
\hline $3-7$ & $0.525072^{+2}$ & $0.134605^{+2}$ & $0.544241^{+2}$ & 0.34 \\
\hline $4-5$ & $0.235759^{+4}$ & $0.819940^{+4}$ & $0.144230^{+5}$ & 1.45 \\
\hline $4-6$ & $0.144916^{+4}$ & $0.594716^{+3}$ & $0.167303^{+4}$ & 0.38 \\
\hline $4-7$ & $0.563801^{+3}$ & $0.147847^{+3}$ & $0.591415^{+3}$ & 0.19 \\
\hline $5-6$ & $0.538081^{+4}$ & $0.329686^{+5}$ & $0.413237^{+5}$ & 1.09 \\
\hline $5-7$ & $0.549144^{+4}$ & $0.215603^{+4}$ & $0.631785^{+4}$ & 0.38 \\
\hline $6-7$ & $0.463205^{+4}$ & $0.104857^{+6}$ & $0.126129^{+6}$ & $1 \cdot 16$ \\
\hline
\end{tabular}

The 10's in the floating point notation have been omitted to save space.

\section{HIGH ENERGY TOTAL INELASTIC CROSS-SECTIONS BY ELECTRON IMPACT}

By making use of the sum rule for the square of matrix elements Inokuti et al. ${ }^{(15)}$ summed formula (10) over all possible excitations and ionizations from the initial term $n l$. The result can be written in a form analogous to (10)

$$
\begin{aligned}
\sigma_{\mathrm{tot}} & =\frac{2}{E}\left(M_{\mathrm{tot}}^{2} \ln c_{\mathrm{tot}}+M_{\mathrm{tot}}^{2} \ln (8 E)\right) . \\
M_{\mathrm{tot}}^{2} & =\frac{n^{2}}{12}\left(n^{2}+3 l^{2}+3 l+11\right) .
\end{aligned}
$$


Its mean value is

$$
\begin{gathered}
\overline{M_{\mathrm{tot}}^{2}}=\frac{n^{2}}{24}\left(5 n^{2}+19\right) . \\
M_{\mathrm{tot}}^{2} \ln c_{\mathrm{tot}}=\alpha^{-2} \int_{\alpha^{-2}}^{\infty} \frac{1-F_{n l}^{2}(x)}{x^{2}} \mathrm{~d} x+\alpha^{-2} \int_{0}^{\alpha^{-2}}\left\{\frac{1-F_{n l}^{2}(x)}{x}-\alpha^{2} M_{\mathrm{tot}}^{2}\right\} \frac{\mathrm{d} x}{x}-2 L(-1) . \\
F_{n l}^{2}(x)=\sum_{l^{\prime}} F_{n l, n l^{\prime}}^{2}(x) . \\
L(-1)=\left\{\int_{n^{\prime} \neq n}\right\} \frac{f_{t}(0)}{2 E_{t}} \ln \left|2 E_{t}\right| .
\end{gathered}
$$

The summation in (16) is over all discrete transitions $t\left(\equiv n l \rightarrow n^{\prime} l^{\prime}\right)$, the integral is over all transitions to the continuum. Only those transitions contribute to (16) for which $l^{\prime}=l \pm 1$. $L(-1)$ can be computed numerically from known analytical expressions for the boundbound and bound-free oscillator strengths (MENZEL and PEKERIs ${ }^{(19)}$ ).

Values of $M_{\text {tot }}^{2}, M_{\text {tot }}^{2} \ln c_{\text {tot }}$, the "discrete" and "continuous" contributions to $L(-1)$ can be found in Table 2 .

TABLE 2. $M_{\mathrm{tot}}^{2}, M_{\mathrm{tot}}^{2} \ln c_{\mathrm{tot}}, L(-1)_{\text {discrete }}$ AND $L(-1)_{\text {continumum }}$

\begin{tabular}{lllll}
\hline Level & $M_{\text {tot }}^{2}$ & $M_{\text {tot }}^{2} \ln c_{\text {tox }}$ & $L(-1)_{\text {discr. }}$ & $L(-1)_{\text {cont. }}$ \\
\hline 1 & 1 & 0.449469 & -0.173168 & +0.0999144 \\
$2 s$ & 5 & 12.05464 & -7.844762 & -0.790892 \\
$2 p$ & 7 & 13.53184 & -12.01771 & -0.573204 \\
3 & 24 & 105.3359 & -65.79481 & -1.717004 \\
4 & 66 & 396.3181 & -232.9429 & -3.037626 \\
5 & 150 & 1100.380 & -623.6760 & -4.528822 \\
6 & 298.5 & 2504.687 & -1396.971 & -5.699587 \\
7 & 539 & 5007.818 & -2763.062 & -7.893913 \\
\hline
\end{tabular}

\section{HIGH ENERGY IONIZATION CROSS-SECTIONS BY ELECTRON IMPACT}

Subtraction of all excitation cross-sections from the total inelastic cross-section gives the ionization cross-section

$$
\sigma_{i}(E)=\sigma_{\mathrm{tot}}(E)-\sum \sigma_{\mathrm{ex}}(E)=\frac{2}{E}\left(M_{i}^{2} \ln c_{i}+M_{i}^{2} \ln (8 E)\right) .
$$

This method has been demonstrated for the He atom by INOKUTI and KIM. ${ }^{(14)}$ As $\sigma_{\text {tot }}$ may contain also de-excitation cross-sections (when a level other than the ground level is considered), these also have to be subtracted in (17). De-excitation cross-sections follow directly from the corresponding excitation cross-sections:

$$
\begin{gathered}
(2 l+1) \sigma_{n l \rightarrow n^{\prime} l^{\prime}}=\left(2 l^{\prime}+1\right) \sigma_{n^{\prime} l^{\prime} \rightarrow n l} \text { or } \\
n^{2} \sigma_{n \rightarrow n^{\prime}}=n^{\prime 2} \sigma_{n^{\prime} \rightarrow n^{\prime}} .
\end{gathered}
$$

As long as only a finite number of excitations are taken into account in (17) $\sigma_{i}$ will exceed the true ionization cross-section. In our calculations $n=7$ represents the highest discrete 
energy level taken into account - the levels $n \geq 8$ are considered to be part of the continuum. It has been shown (MAHN ${ }^{(18)}$ ) that this is the proper way to treat the ionization of a hydrogen-like ion in this model.

By means of the transformations

$$
\begin{aligned}
& a=4 M_{i}^{2}\left(1 / n^{2}-1 / 64\right)^{-1} \\
& b=a \ln 4 c_{i}\left(1 / n^{2}-1 / 64\right)
\end{aligned}
$$

we obtain the ionization cross-section in the form

$$
\sigma_{i}(U)=\frac{1}{U}(a+b \ln (U)) .
$$

We recall that in this model the threshold energy for ionization from level $n$ is

$$
E_{t}=\frac{1}{2} Z^{2}\left(1 / n^{2}-1 / 64\right)
$$

Values of $M_{i}^{2}, M_{i}^{2} \ln c_{i}, a$ and $b$ for ionization can be found in Table 3. As a comparison the values for true ionization as computed by VRIENS and BONSEN ${ }^{(26)}$ are included in Table 3.

TABLE 3. $a, b, c, p, M_{i}^{2}$ AND $M_{i}^{2} \ln c_{i}$ FOR IONIZATION. (1) $M_{i}^{2}$ AND $M_{i}^{2} \ln c_{i}$ FOR "TRUE" IONIZATION ACCORDING TO INOKUTI. ${ }^{(13)}(2) M_{i}^{2}$ AND $M_{i}^{2} \ln c_{i}$ FOR "TRUE" IONIZATION ACCORDING TO VRIENS AND BONSEN ${ }^{(26)}$

\begin{tabular}{lcccccc}
\hline Level & $a$ & $b$ & $c$ & $M_{i}^{2}$ & $M_{i}^{2} \ln c_{i}$ & $p$ \\
\hline 1 & $0.517113^{1}$ & $0.120971^{1}$ & $0.195^{-1}$ & $0.297702^{0}$ & $0.864589^{0}$ & 1.35 \\
$(1)$ & & & $0.283412^{0}$ & $0.863668^{0}$ & \\
$2 s$ & $0.816565^{2}$ & $0.165274^{2}$ & $0.159^{1}$ & $0.968400^{0}$ & $0.484707^{1}$ & 0.919 \\
$(2 s)$ & & & & $0.823193^{0}$ & $0.44974^{1}$ & \\
$2 p$ & $0.107561^{3}$ & $0.114219^{2}$ & $0.190^{1}$ & $0.669254^{0}$ & $0.634558^{1}$ & 0.944 \\
$(2 p)$ & & & & $0.531166^{0}$ & $0.58377^{1}$ & \\
3 & $0.228230^{4}$ & $0.638291^{2}$ & $0.308^{2}$ & $0.152370^{1}$ & $0.559484^{2}$ & 2.67 \\
4 & $0.183315^{5}$ & $0.277747^{3}$ & $0.287^{3}$ & $0.325485^{1}$ & $0.220270^{3}$ & 3.92 \\
5 & $0.102446^{6}$ & $0.137642^{4}$ & $0.220^{4}$ & $0.838756^{1}$ & $0.643805^{3}$ & 4.30 \\
6 & $0.487943^{6}$ & $0.108225^{5}$ & $0.188^{5}$ & $0.328808^{2}$ & $0.158189^{4}$ & 3.30 \\
7 & $0.244476^{7}$ & $0.327071^{6}$ & $0.325^{6}$ & $0.391108^{3}$ & $0.447078^{4}$ & 1.25 \\
\hline
\end{tabular}

\section{INTERPOLATION OF THE EXCITATION AND IONIZATION CROSS-SECTIONS BETWEEN THRESHOLD AND HIGH ENERGIES}

Excitation cross-sections in the Coulomb-Born approximation (or higher approximations) can be calculated only in a narrow energy range near threshold (as long as the convenient but slowly converging coupled angular momentum expansion remains the only practical method for computing the matrix elements of electrostatic interaction; $\mathrm{JACOBS}^{(16)}$ ). On the other hand the Bethe-Born approximation is reliable only for very high energies, the lower limit of which is still a matter of discussion.

In the Bethe-Born approximation $\sigma \times U$ is a linear function of $\ln U$. We assume (9) and (20) to be valid for $\ln U \geq p$, where $p$ is a positive number. Our interpolation assumes a linear relationship between $\sigma U$ and $\ln U$ to hold also for $0 \leq \ln U \leq p$ under the conditions that at $U=1$ the cross-section has the calculated C.B. value $c\left(\mathrm{JACOBS}^{(16)}\right)$ and that in 
$U=\mathrm{e}^{p}$ the cross-section is continuous. Accordingly the formula for the cross-section valid at all energies will be

$$
\sigma=\frac{1}{U}\left[c+\frac{a-c+b p}{p} \ln U+\mu\left(U-\mathrm{e}^{p}\right)\left\{a-c+\frac{c-a}{p} \ln U\right\}\right]
$$

$c=\sigma(1)$.

$$
\left.\mu(x) \text {, the unit step function }=\begin{array}{l}
1 \text { for } x \geq 0 \\
0 \text { for } x<0
\end{array}\right\} .
$$

In (22) $p$ is a yet undetermined parameter. It is clear that $\sigma$ will be practically independent on $p$ when $|a-c| \ll b$. Fortunately this condition is fulfilled for all transitions for which $n^{\prime} \geq n+2$ and for $1 s \rightarrow 2 s$.

A value can be assigned to $p$ when an experimental or theoretical value of $\sigma$ at an intermediate energy $(1<U \leqslant 10)$ is available. We computed the first derivative of $\sigma$ with respect to $U$ in the C.B. approximation at threshold

$$
c^{\prime} \equiv\left(\frac{\mathrm{d} \sigma}{\mathrm{d} U}\right)_{U=1} \approx 1000(\sigma(1 \cdot 001)-\sigma(1)) .
$$

From (22) it follows that

$$
p=\frac{a-c}{c^{\prime}+c-b} .
$$

Whereas $c$ and $c^{\prime}$ for excitations can be obtained directly in the Coulomb-Born approximation, their values for ionization have to be computed in a different less exact way. In our model the threshold value of the ionization cross-section equals the excitation cross-section at threshold to the level $n^{\prime}=8$. This value can be estimated by means of the empirical formula

$$
c_{n, 8}=9.53 n^{19 / 3} 8^{-3}\left(1-\frac{n^{2}}{64}\right)^{-3} .
$$

(cf. Jacobs, formula 31). $c^{\prime}$ can be derived from the same formula

$$
c^{\prime}=256\left(1 / n^{2}-1 / 64\right) c \text {. }
$$

Values of $c$ and $p$ for excitation and ionization can be found in Tables 1 and 3 respectively.

\section{EXCITATION AND IONIZATION CROSS-SECTIONS BY PROTON IMPACT}

The Born-Bethe formula for excitation and ionization by protons is obtained from (9) or (20) substituting $U / M$ instead of $U$.

$$
\sigma^{p r}=\frac{M}{U}(a+b \ln (U / M)) .
$$

$M$ is the proton mass, 1836.12 atomic mass units. At threshold the cross-sections and all their derivatives vanish due to the repulsive Coulomb field. The wave function of the proton 
at low velocities $v$ is proportional to $\mathrm{e}^{-z / v}$. The threshold behaviour of the cross-section will be roughly proportional to $U^{-1}$ times the square of the product of the proton wavefunctions before and after the collision.

$$
\sigma^{p r} \sim \frac{1}{U} \exp \left[-d\left(\frac{1}{\sqrt{ }(U-1)}+\frac{1}{\sqrt{ }(U)}\right)\right]
$$

where

$$
d=\frac{2 \pi \sqrt{ }(M)}{t}
$$

and $t$ has been defined in Section 2 .

In order to construct an expression for the cross-sections by proton impact we assume (27) to be true for $1 \leq U \leq \beta$ and choose the constant of proportionality in (27) so that $\sigma(U)$ will be differentiable for all $U>1$. For $U \geq \beta(26)$ should hold. We define

$$
\alpha=\ln \beta-\ln M+a / b
$$

so that (26) may be written

$$
\sigma=\frac{M b}{U}(\alpha+\ln (U / \beta)) \quad(U \geq \beta)
$$

The constant of proportionality in (27) is found by equating the value and the first derivative of $\sigma$ in $U=\beta$ in (30) and (27):

$$
\sigma^{p r}(U)=\frac{M b}{U} \alpha \exp \left[-d\left\{\frac{1}{\sqrt{ } U}+\frac{1}{\sqrt{ }(U-1)}-\frac{1}{\sqrt{ } \beta}-\frac{1}{\sqrt{ }(\beta-1)}\right], \quad(U \leq \beta) .\right.
$$

$\alpha$ must be calculated from the implicit expression

$$
\alpha=\ln \beta-\ln M+a / b=\frac{2 \sqrt{ } \beta}{d\left\{1+[\beta /(\beta-1)]^{3 / 2}\right\}} .
$$

Proton excitation cross-sections for $1 s \rightarrow 2 s$ and other non-dipole transitions cannot be calculated in this way because $b=0$. However, we can subtract the $1 s \rightarrow 2 p$ cross-section from the $1 s \rightarrow(2 s+2 p)$ cross-section.

Values of $\alpha, \beta$ and $d$ can be found in Table 4 .

\section{RATE COEFFICIENTS FOR EXCITATION AND IONIZATION}

The rate coefficient $C_{t}$ for the process (transition) $t$ is the time average of the crosssection of that process times the velocity of the incident particle taken over a period of time in which the mean kinetic energy of the particles $\bar{E}$ does not change appreciably. The rate coefficient measures the number of processes $t$ in unit time and unit concentrations of scattering and scattered particles. In a thermal (Maxwellian) plasma the expression for the rate coefficient is

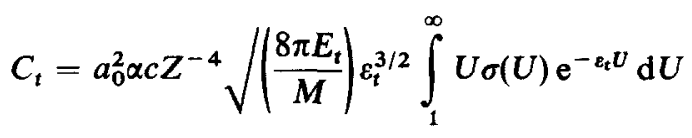


TABLE 4. $\alpha, \beta, d$ FOR PROTON IMPACT IONIZATION AND EXCITATION

\begin{tabular}{|c|c|c|c|c|c|c|c|}
\hline Transition & $\alpha$ & $\beta$ & $d$ & Transition & $\alpha$ & $\boldsymbol{\beta}$ & $d$ \\
\hline $1-(2 s+2 p)$ & $0.120^{-0}$ & $0.139^{+4}$ & $0.311^{+3}$ & $3-4$ & $0.290^{-1}$ & $0.126^{+4}$ & $0.122^{+4}$ \\
\hline $1-2 p$ & $0.133^{-0}$ & $0.171^{+4}$ & $0.311^{+3}$ & $3-5$ & $0.141^{-1}$ & $0.203^{+3}$ & $0.101^{+4}$ \\
\hline $1-3$ & $0.956^{-1}$ & $0.746^{+3}$ & $0.286^{+3}$ & $3-6$ & $0.898^{-2}$ & $0.717^{+2}$ & $0.933^{+3}$ \\
\hline $1-4$ & $0.880^{-1}$ & $0.600^{+3}$ & $0.278^{+3}$ & $3-7$ & $0.670^{-2}$ & $0.374^{+2}$ & $0.894^{+3}$ \\
\hline $1-5$ & $0.846^{-1}$ & $0.542^{+3}$ & $0.275^{+3}$ & 3-ion & $0.496^{+2}$ & $0.187^{+10}$ & $0.871^{+3}$ \\
\hline $1-6$ & $0.828^{-1}$ & $0.513^{+3}$ & $0.273^{+3}$ & & & & \\
\hline $1-7$ & $0.818^{-1}$ & $0.496^{+3}$ & $0.272^{+3}$ & $4-5$ & $0.209^{-1}$ & $0.141^{+4}$ & $0.179^{+4}$ \\
\hline \multirow[t]{2}{*}{ 1-ion } & $0.182^{-1}$ & $0.260^{+2}$ & $0.271^{+3}$ & $4-6$ & $0.877^{-2}$ & $0.161^{+3}$ & $0.144^{+4}$ \\
\hline & & & & $4-7$ & $0-477^{-2}$ & $0.407^{+2}$ & $0.131^{+4}$ \\
\hline $2 s-3$ & $0.349^{-1}$ & $0.639^{+3}$ & $0.722^{+3}$ & 4-ion & $0.815^{+2}$ & $0.103^{+11}$ & $0.124^{+4}$ \\
\hline $2 s-4$ & $0.304^{-1}$ & $0.359^{+3}$ & $0.622+3$ & & & & \\
\hline $2 s-5$ & $0-278^{-1}$ & $0.267^{+3}$ & $0.588^{+3}$ & $5-6$ & $0.163^{-1}$ & $0.159^{+4}$ & $0.244^{+4}$ \\
\hline $2 s-6$ & $0.263^{-1}$ & $0.227^{+3}$ & $0.571^{+3}$ & $5-7$ & $0.622^{-2}$ & $0.145^{+3}$ & $0.192^{+4}$ \\
\hline $2 s-7$ & $0.254^{-1}$ & $0.205^{+3}$ & $0.562^{+3}$ & 5-ion & $0.908^{+2}$ & $0.245^{+11}$ & $0.172^{+4}$ \\
\hline \multirow[t]{2}{*}{$2 s$-ion } & $0.615^{-2}$ & $0.132^{+2}$ & $0.556^{+3}$ & & & & \\
\hline & & & & $6-7$ & $0.134^{-1}$ & $0.178^{+4}$ & $0.314^{+4}$ \\
\hline $2 p-3$ & $0.507^{-1}$ & $0.134^{+4}$ & $0.722^{+3}$ & 6-ion & $0.614^{+2}$ & $0.225^{+11}$ & $0.244^{+4}$ \\
\hline $2 p-4$ & $0.280^{-1}$ & $0.304^{+3}$ & $0.622^{+3}$ & & & & \\
\hline $2 p-5$ & $0.204^{-1}$ & $0.145^{+3}$ & $0.588^{+3}$ & 7-ion & $0.415^{-5}$ & $0.104^{+1}$ & $0.389^{+4}$ \\
\hline $2 p-6$ & $0.169^{-1}$ & $0.949^{+2}$ & $0.571^{+3}$ & & & & \\
\hline $2 p-7$ & $0.151^{-1}$ & $0.732^{+2}$ & $0.562^{+3}$ & & & & \\
\hline $2 p$-ion & $0.206^{+2}$ & $0.131^{+9}$ & $0.556^{+3}$ & & & & \\
\hline
\end{tabular}

where

$$
\begin{aligned}
a_{0}^{2} \alpha c \sqrt{ }(8 \pi) & =3.071116810^{-8} \mathrm{~cm}^{3} \mathrm{sec}^{-1} \\
T & =\frac{2 \bar{E}}{3 k} \text { (the } T \text { of electrons, protons, ions etc. need not be equal) } \\
k & =3.16675610^{-6} \text { atomic energy units } /{ }^{\circ} \mathrm{K} \\
\varepsilon_{t} & =E_{t} /(k T)
\end{aligned}
$$

$M$ is the mass of the scattered particle in atomic units (electron rest mass).

Substitution of (22) into (33) gives for incident electrons $(M=1)$

$$
C=3.0710^{-8} \frac{\varepsilon \sqrt{ }(k T)}{Z^{4}}\left[c \mathrm{e}^{-\varepsilon}+\left(c+c^{\prime}\right) E_{1}(\varepsilon)-\left(c+c^{\prime}-b\right) E_{1}\left(\varepsilon \mathrm{e}^{p}\right)\right] \mathrm{cm}^{3} \sec ^{-1} .
$$

Here we have used our choice (23) of $p$ and omitted the suffix $t . E_{1}$ is the exponential integral function. The rate coefficient for incident protons is obtained by substituting (30) and (31) into (33). The resulting expression can be evaluated by means of a numerical integration. For low relative temperatures $(\varepsilon \gg 1)$ the rate coefficients for proton impact are much smaller than the rate coefficients for electron impact and therefore may be neglected in plasmas consisting mainly of electrons.

As $c$ and $c^{\prime}$ are slowly varying functions of $Z\left(\mathrm{JACOBS}^{(16)}\right.$ and $\varepsilon_{t}=Z^{2} /\left(2 t^{2} k T\right)$ it follows from (34) that $Z^{3} C_{t}$ as a function of $T / Z^{2}$ is almost independent of $Z$. In Table 5 we give the scaled rate coefficients $Z^{3} C$ for a few transitions and a few scaled temperatures $T / Z^{2}$ for electron impact. Proton impact rate coefficients can be found in Table 6 . The values for $Z \rightarrow \infty$ of $c$ and $c^{\prime}$ have been used. 
TABLE 5. $Z^{3} \times$ RATE COEFFICIENTS FOR A FEW EXCITATIONS AND IONIZATIONS AS FUNCTIONS OF $T / Z^{2}$ IN UNITS $\mathrm{cm}^{3} \mathrm{sec}^{-1}$. ELECTRON IMPACT

\begin{tabular}{|c|c|c|c|c|c|c|c|c|}
\hline$T / Z^{2}$ & $1-2 s$ & $1-2 p$ & $1-3$ & 1-ion & $2 s-3$ & $2 s$-ion & $2 p-3$ & $2 p$-ion \\
\hline $1.010^{3}$ & $0.442^{-58}$ & $0.176^{-57}$ & $0.139^{-67}$ & $0.439^{-75}$ & $0.171^{-14}$ & $0.235^{-22}$ & $0.218^{-14}$ & $0.279^{-22}$ \\
\hline $2.010^{3}$ & $0.162^{-32}$ & $0.648^{-32}$ & $0.295^{-37}$ & $0.281^{-41}$ & $0.708^{-10}$ & $0.288^{-14}$ & $0.899^{-10}$ & $0.343^{-14}$ \\
\hline $3.010^{3}$ & $0.493^{-24}$ & $0-198^{-23}$ & $0.348^{-27}$ & $0.562^{-30}$ & $0.226^{-8}$ & $0.153^{-11}$ & $0.286^{-8}$ & $0.182^{-11}$ \\
\hline $4.010^{3}$ & $0.825^{-20}$ & $0.333^{-19}$ & $0.363^{-22}$ & $0.260^{-24}$ & $0.123^{-7}$ & $0.362^{-10}$ & $0.155^{-7}$ & $0.431^{-10}$ \\
\hline $5.010^{3}$ & $0.275^{-17}$ & $0.111^{-16}$ & $0.364^{-19}$ & $0.668^{-21}$ & $0.333^{-7}$ & $0.246^{-9}$ & $0.418^{-7}$ & $0.293^{-9}$ \\
\hline $6.010^{3}$ & $0.130^{-15}$ & $0.530^{-15}$ & $0.359^{-17}$ & $0.127^{-18}$ & $0.636^{-7}$ & $0.891^{-9}$ & $0.799^{-7}$ & $0.106^{-8}$ \\
\hline $8.010^{3}$ & $0.157^{-13}$ & $0.643^{-13}$ & $0 \cdot 108^{-14}$ & $0.921^{-16}$ & $0.140^{-6}$ & $0.454^{-8}$ & $0.175^{-6}$ & $0.541^{-8}$ \\
\hline $1.010^{4}$ & $0.271^{-12}$ & $0.112^{-11}$ & $0.326^{-13}$ & $0-489^{-14}$ & $0.220^{-6}$ & $0.122^{-7}$ & $0.273^{-6}$ & $0.145^{-7}$ \\
\hline $1.210^{4}$ & $0.178^{-11}$ & $0.743^{-11}$ & $0.310^{-12}$ & $0.670^{-13}$ & $0.293^{-6}$ & $0.237^{-7}$ & $0.363^{-6}$ & $0.282^{-7}$ \\
\hline $1.610^{4}$ & $0.182^{-10}$ & $0.770^{-10}$ & $0.506^{-11}$ & $0.199^{-11}$ & $0.412^{-6}$ & $0.548^{-7}$ & $0.506^{-6}$ & $0.653^{-7}$ \\
\hline $2.010^{4}$ & $0.719^{-10}$ & $0.307^{-9}$ & $0.265^{-10}$ & $0.151^{-10}$ & $0.496^{-6}$ & $0.907^{-7}$ & $0.607^{-6}$ & $0.108^{-6}$ \\
\hline $2.510^{4}$ & $0.211^{-9}$ & $0.915^{-9}$ & $0.977^{-10}$ & $0.778^{-10}$ & $0.569^{-6}$ & $0.135^{-6}$ & $0.692^{-6}$ & $0.161^{-6}$ \\
\hline $3.010^{4}$ & $0.424^{-9}$ & $0.187^{-8}$ & $0.230^{-9}$ & $0.234^{-9}$ & $0.617^{-6}$ & $0.175^{-6}$ & $0.749^{-6}$ & $0.209^{-6}$ \\
\hline $4.010^{4}$ & $0.990^{-9}$ & $0.448^{-8}$ & $0.657^{-9}$ & $0.942^{-9}$ & $0.673^{-6}$ & $0.240^{-6}$ & $0.814^{-6}$ & $0.285^{-6}$ \\
\hline $5.010^{4}$ & $0.161^{-8}$ & $0.745^{-8}$ & $0.121^{-8}$ & $0.220^{-8}$ & $0.701^{-6}$ & $0.285^{-6}$ & $0.848^{-6}$ & $0.338^{-\kappa}$ \\
\hline $6.010^{4}$ & $0.218^{-8}$ & $0.104^{-7}$ & $0.180^{-8}$ & $0.390^{-8}$ & $0.714^{-6}$ & $0.316^{-6}$ & $0.866^{-6}$ & $0.374^{-6}$ \\
\hline $8.010^{4}$ & $0.311^{-8}$ & $0.154^{-7}$ & $0.291^{-8}$ & $0.805^{-8}$ & $0.721^{-6}$ & $0.352^{-6}$ & $0.881^{-6}$ & $0.416^{-6}$ \\
\hline $1.010^{5}$ & $0.375^{-8}$ & $0.193^{-7}$ & $0.382^{-8}$ & $0.125^{-7}$ & $0.717^{-6}$ & $0.369^{-6}$ & $0.883^{-6}$ & $0.434^{-6}$ \\
\hline $1.210^{5}$ & $0.418^{-8}$ & $0.224^{-7}$ & $0.455^{-8}$ & $0.168^{-7}$ & $0.708^{-6}$ & $0.377^{-6}$ & $0.879^{-6}$ & $0.440^{-6}$ \\
\hline $1.610^{5}$ & $0.464^{-8}$ & $0.266^{-7}$ & $0.558^{-8}$ & $0.243^{-7}$ & $0.688^{-6}$ & $0.377^{-6}$ & $0.865^{-6}$ & $0.437^{-6}$ \\
\hline $2.010^{5}$ & $0.481^{-8}$ & $0.294^{-7}$ & $0.624^{-8}$ & $0.301^{-7}$ & $0.667^{-6}$ & $0.370^{-6}$ & $0.849^{-6}$ & $0.426^{-6}$ \\
\hline $2.510^{5}$ & $0.484^{-8}$ & $0.317^{-7}$ & $0.676^{-8}$ & $0.354^{-7}$ & $0.644^{-6}$ & $0.359^{-6}$ & $0.828^{-6}$ & $0.410^{-6}$ \\
\hline $3.010^{5}$ & $0.478^{-8}$ & $0.332^{-7}$ & $0.709^{-8}$ & $0.392^{-7}$ & $0.623^{-6}$ & $0.347^{-6}$ & $0.808^{-6}$ & $0.393^{-6}$ \\
\hline $4.010^{5}$ & $0.456^{-8}$ & $0.349^{-7}$ & $0.742^{-8}$ & $0.437^{-7}$ & $0.587^{-6}$ & $0.325^{-6}$ & $0.773^{-6}$ & $0.365^{-6}$ \\
\hline $5.010^{5}$ & $0.432^{-8}$ & $0.358^{-7}$ & $0.755^{-8}$ & $0.459^{-7}$ & $0.559^{-6}$ & $0.306^{-6}$ & $0.743^{-6}$ & $0.341^{-6}$ \\
\hline
\end{tabular}

\section{THE $2 s-2 p$ TRANSITIONS}

The collision induced transitions $2 s-2 p$ are the most efficient process quenching the $2 s-1 s$ two-photon spontaneous radiation and compete with the latter process in depopulating the $2 s$ state.

The treatment of the $2 s-2 p$ transitions differs in two aspects from the $n \neq n^{\prime}$ transitions. Firstly, the threshold energies are very much smaller so that $\varepsilon$ is always much smaller than

TABLE 6. $Z^{3} \times$ RATE COEFFICIENTS FOR A FEW EXCITATIONS AND IONIZATIONS AS FUNCTIONS OF $T / Z^{2}$ IN UNITS $\mathrm{cm}^{3} \mathrm{sec}^{-1}$. Proton IMPaCt

\begin{tabular}{|c|c|c|c|c|c|c|c|c|}
\hline$T / Z^{2}$ & $1-2 s$ & $1-2 p$ & $1-3$ & 1-ion & $2 s-3$ & $2 s$-ion & $2 p-3$ & $2 p$-ion \\
\hline $6.010^{4}$ & $0.792^{-95}$ & $0.132^{-96}$ & $0.560^{-92}$ & $0.313^{-36}$ & $0.106^{-79}$ & $0.197^{-9}$ & $0.686^{-81}$ & $0.362^{-120}$ \\
\hline $8.010^{4}$ & $0.108^{-80}$ & $0.853^{-82}$ & $0.327^{-79}$ & $0.648^{-29}$ & $0.534^{-73}$ & $0.168^{-8}$ & $0.124^{-72}$ & $0.312^{-97}$ \\
\hline $1.010^{5}$ & $0.280^{-72}$ & $0.607^{-73}$ & $0.139^{-71}$ & $0.161^{-24}$ & $0.982^{-67}$ & $0.614^{-8}$ & $0.107^{-67}$ & $0.167^{-83}$ \\
\hline $1.210^{5}$ & $0.965^{-67}$ & $0.460^{-67}$ & $0.160^{-63}$ & $0.138^{-21}$ & $0.122^{-56}$ & $0.147^{-7}$ & $0.347^{-64}$ & $0.225^{-74}$ \\
\hline $1.610^{5}$ & $0.443^{-60}$ & $0.942^{-60}$ & $0.312^{-60}$ & $0.652^{-18}$ & $0.525^{-44}$ & $0.446^{-7}$ & $0.106^{-58}$ & $0.533^{-63}$ \\
\hline $2 \cdot 010^{5}$ & $0.124^{-56}$ & $0.213^{-55}$ & $0.178^{-56}$ & $0 \cdot 106^{-15}$ & $0.199^{-36}$ & $0.876^{-7}$ & $0.622^{-55}$ & $0.330^{-56}$ \\
\hline $2.510^{5}$ & $0.184^{-52}$ & $0.617^{-52}$ & $0.428^{-53}$ & $0.635^{-14}$ & $0.230^{-30}$ & $0.151^{-6}$ & $0.227^{-51}$ & $0.836^{-51}$ \\
\hline $3.010^{5}$ & $0.865^{-51}$ & $0.181^{-49}$ & $0.330^{-50}$ & $0.983^{-13}$ & $0.253^{-26}$ & $0.219^{-6}$ & $0.296^{-48}$ & $0.319^{-47}$ \\
\hline $4.010^{5}$ & $0.190^{-46}$ & $0.278^{-45}$ & $0.256^{-46}$ & $0.308^{-11}$ & $0.287^{-21}$ & $0.351^{-6}$ & $0.781^{-38}$ & $0.872^{-43}$ \\
\hline $5-010^{5}$ & $0.339^{-43}$ & $0.160^{-42}$ & $0.170^{-43}$ & $0.247^{-10}$ & $0.313^{-18}$ & $0-466^{-6}$ & $0.184^{-31}$ & $0.373^{-40}$ \\
\hline
\end{tabular}


unity and therefore only the $U \gg 1$ limit of the cross-sections are important and, secondly, the $Z$-dependence of the threshold energies is different. $Z \geq 2$ )

We distinguish between the $2^{2} P_{1 / 2}$ and the $2^{2} P_{3 / 2}$ terms. The transition energies are (for

$$
\begin{aligned}
& \Delta_{1 / 2}=-1.7910^{-7} Z^{4} \\
& \Delta_{3 / 2}=1.5310^{-6} Z^{4} \text { atomic units. }
\end{aligned}
$$

We shall always take the absolute value of $\Delta_{1 / 2}$.

The function $F^{2}(x)$ for $1 s-2 p$ is

and

$$
F^{2}(x)=(x+1)^{-8}\left(9 x-18 x^{2}+9 x^{3}\right)
$$

$$
\alpha=1 \text {. }
$$

$t^{2}$ is meaningless in this case but we may still define

$$
U=E / \Delta
$$

and

$$
\varepsilon=\Delta / k T
$$

$F^{2}(x)$ in (36) is the mean function for all $2 s-2 p$ transitions. For the transitions to $j=1 / 2$ and $j=3 / 2$ we have to multiply the right-hand side of (36) by weights $\omega$ which are $1 / 3$ and $2 / 3$ respectively. The Bethe-Born formula for the cross-sections are derived in the same manner as (8).

$$
\sigma=\omega \frac{\pi a_{0}^{2} M}{Z^{2} \Delta U} 18\left[\ln \frac{2 Z^{2}}{\Delta M}-\frac{1199}{420}+\ln U\right] .
$$

We write (37) in the form (9) by means of the definitions

$$
b=\omega 18 Z^{2} M / \Delta
$$

and

$$
a=b\left[\ln \frac{2 Z^{2}}{\Delta M}-1199 / 420\right] .
$$

As $\Delta \sim Z^{4} a$ and $b$ still depend on $Z$ contrary to the $n \neq n^{\prime}$ transitions. We retain $M$ in all expressions. $M$ should be taken as unity for transitions caused by electrons.

As in most physical applications $1 / \varepsilon \gg 1$, the threshold behaviour of $\sigma$ is not important. We therefore suppose (37) to be true for all values of $U \geq 1$. In those cases where $a<0$ (for $M=1836.12$ and $Z>6$ ) we take $\sigma=0$ for $\ln U \leq-a / b$ in order to avoid negative values of $\sigma$.

Application of (33) gives the rate coefficients

$$
\begin{array}{ll}
C=\frac{a_{0}^{2} \alpha c}{Z^{2}} \sqrt{ }\left(\frac{8 \pi M}{k T}\right) \omega 18\left[\frac{a}{b} \mathrm{e}^{-\varepsilon}+E_{1}(\varepsilon)\right] & \text { for } a>0 \\
C=\frac{a_{0}^{2} \alpha c}{Z^{2}} \sqrt{ }\left(\frac{8 \pi M}{k T}\right) \omega 18 E_{1}\left(\varepsilon \mathrm{e}^{-a / b}\right) & \text { for } a \leq 0 .
\end{array}
$$


For the transition to $2 P_{1 / 2}$ we must also multiply these expressions by $\mathrm{e}^{\varepsilon}$ because it is a deexcitation. (This follows from the principle of detailed balancing. (39) and (40) actually give the rate of the inverse process but for the ratio of the statistical weights.)

We expand (39) and (40) in ascending power series in $\varepsilon$ and retain only the constant and the logarithmic terms in $\varepsilon$.

$$
C \approx \frac{a_{0}^{2} \alpha c}{Z^{2}} \sqrt{\left(\frac{8 \pi M}{k T}\right) 18 \omega(a / b-0.57722-\ln \varepsilon) .}
$$

This approximation is valid as long as $\varepsilon$ can be neglected against $\ln 1 / \varepsilon$. Substitution of (35) and (38) into (41) shows that $C Z^{5}$ as function of $T / Z^{6}$ is independent on $Z$. Values of $C Z^{5}$ can be found in Table 7 .

TABLE 7. $Z^{5}$ RATE COEFFICIENTS FOR THE TRANSITIONS $2 S_{1 / 2}-2 P_{1 / 2}$ AND $2 S_{1 / 2}-2 P_{3 / 2}$ BY ELECTRON AND PROTON IMPACT AS FUNCTIONS OF $T / Z^{6}$

\begin{tabular}{|c|c|c|c|c|}
\hline & \multicolumn{2}{|c|}{ Electrons } & \multicolumn{2}{|c|}{ Protons } \\
\hline$T / Z^{6}$ & $1 / 2-1 / 2$ & $1 / 2-3 / 2$ & $1 / 2-1 / 2$ & $1 / 2-3 / 2$ \\
\hline 1 & $0.162^{-2}$ & $0.236^{-2}$ & $0.362^{-1}$ & $0.343^{-1}$ \\
\hline 2 & $0.120^{-2}$ & $0.177^{-2}$ & $0.278^{-1}$ & $0.286^{-1}$ \\
\hline 3 & $0.100^{-2}$ & $0.149^{-2}$ & $0.237^{-1}$ & $0.254^{-1}$ \\
\hline 4 & $0.883^{-3}$ & $0.132^{-2}$ & $0.212^{-1}$ & $0.233^{-1}$ \\
\hline 5 & $0.800^{-3}$ & $0.120^{-2}$ & $0.194^{-1}$ & $0.217^{-1}$ \\
\hline 6 & $0.738^{-3}$ & $0.111^{-2}$ & $0.180^{-1}$ & $0.205^{-1}$ \\
\hline 7 & $0.689^{-3}$ & $0.104^{-2}$ & $0.169^{-1}$ & $0.196^{-1}$ \\
\hline 8 & $0.650^{-3}$ & $0.985^{-3}$ & $0.160^{-1}$ & $0.186^{-1}$ \\
\hline 9 & $0.617^{-3}$ & $0.937^{-3}$ & $0.153^{-1}$ & $0.179^{-1}$ \\
\hline 10 & $0.588^{-3}$ & $0.896^{-3}$ & $0.147^{-1}$ & $0.173^{-1}$ \\
\hline 20 & $0.432^{-3}$ & $0-666^{-3}$ & $0.111^{-1}$ & $0.136^{-1}$ \\
\hline 30 & $0.360^{-3}$ & $0.559^{-3}$ & $0.936^{-2}$ & $0.118^{-1}$ \\
\hline 40 & $0.317^{-3}$ & $0.493^{-3}$ & $0.831^{-2}$ & $0.106^{-1}$ \\
\hline 50 & $0.287^{-3}$ & $0.448^{-3}$ & $0.757^{-2}$ & $0.976^{-2}$ \\
\hline 60 & $0.264^{-3}$ & $0.414^{-3}$ & $0.702^{-2}$ & $0.912^{-2}$ \\
\hline 70 & $0.246^{-3}$ & $0.387^{-3}$ & $0.658^{-2}$ & $0.860^{-2}$ \\
\hline 80 & $0.232^{-3}$ & $0.365^{-3}$ & $0.622^{-2}$ & $0.818^{-2}$ \\
\hline 90 & $0.220^{-3}$ & $0.347^{-3}$ & $0.592^{-2}$ & $0.782^{-2}$ \\
\hline 100 & $0.210^{-3}$ & $0.331^{-3}$ & $0.566^{-2}$ & $0.751^{-2}$ \\
\hline
\end{tabular}

This table serves only as an illustration and should not be used when accurate values are required. The main error introduced is taking 1836 for the mass of the proton instead of the reduced mass.

\section{COMPARISON WITH OTHER FORMULAE}

We shall compare formula (34) with the most widely used expressions for excitation and ionization rate coefficients. With the exception of (43a) these expressions are explicitly meant to be applicable only for low temperatures. By "low" temperatures we mean that $E_{1}(\varepsilon) \approx$ $\varepsilon^{-1} \mathrm{e}^{-\varepsilon}$. (It is regrettable that in a large number of publications on ionization equilibria formula $44 a$ has been used outside its range of applicability.) 
We shall first derive the low temperature limit of (34). The third term between the square brackets may be neglected, because for $p \gg 0$ the exponential integral will be very small and for $p=0$ it follows from (22) that $c=a$ and $c^{\prime}=b-a$, so that $c+c^{\prime}-b=0$. Of the second term we replace the exponential integral by the first term of its asymptotical expansion.

$$
C=3.0710^{-8} \frac{\varepsilon \sqrt{ }(k T)}{Z^{4}}\left[c \mathrm{e}^{-\varepsilon}+\frac{c^{\prime}+c}{\varepsilon} \mathrm{e}^{-\varepsilon}\right] .
$$

(All rate coefficients are expressed in $\mathrm{cm}^{3} \mathrm{sec}^{-1}$ ). For excitation $c \neq 0$ and we shall neglect the second term in (42). For ("true") ionization $c=0$ and we retain only the second term. We have for excitation

$$
C_{\mathrm{ex}}=3.0710^{-8} \frac{c \sqrt{ }(k T)}{Z^{4}} \varepsilon \mathrm{e}^{-\varepsilon}
$$

For ionization

$$
C_{\text {ion }}=3.0710^{-8} \frac{c^{\prime} \sqrt{ }(k T)}{Z^{4}} \mathrm{e}^{-\varepsilon}
$$

The following expressions for $C_{\mathrm{ex}}$ and $C_{\mathrm{ion}}$ have been used most widely:

$$
\begin{array}{ll}
C_{\text {ex }}=11.110^{-8} \frac{b \sqrt{ }(k T)}{Z^{4}} \varepsilon \mathrm{e}^{-\varepsilon} P(\varepsilon) & \text { SEATON }^{(24)} \\
C_{\text {ex }}=A 10^{-8} \frac{\sqrt{ }(k T)}{Z^{4}} \varepsilon \mathrm{e}^{-\varepsilon} \frac{\varepsilon+1}{\varepsilon+\chi} & \text { BeIGMAN } \text { et al. }{ }^{(1)} \\
C_{\text {ion }}=6.110^{-8} \frac{n^{4} \sqrt{ }(k T)}{Z^{4}} \mathrm{e}^{-\varepsilon} & \text { BURGESS and SEATON }{ }^{(9)} \\
C_{\text {ion }}=A 10^{-8} \frac{\sqrt{ }(k T)}{Z^{4}} \mathrm{e}^{-\varepsilon} \frac{\varepsilon}{\varepsilon+\chi} & \text { BeIGMAN } \text { et al. }{ }^{(1)} \\
C_{\text {ion }}=3.4810^{-8}\left(3.1-\frac{1.2}{Z}-\frac{0.9}{Z^{2}}\right) \frac{n^{4}}{Z^{4}} \sqrt{ }(k T) \mathrm{e}^{-\varepsilon} . & \text { BuRGESS } \text { et al. } .^{(7)}
\end{array}
$$

We have adapted all quoted formulae to our notation and system of units. Moreover, in (43b) and (44b) we have assimilated into $A$ all other transition-dependent constants, so that $A$ is numerically not the same as in formula (2) of the original article by BEIGMAN $e t$ al..$^{(1)}$

The function $P(\varepsilon)$ in (43a) has been tabulated by VAN REGEMORTER. ${ }^{(22)}$ For $\varepsilon \gg 1, P(\varepsilon) \approx$ 0.2. Comparing with (43) shows that Seaton's excitation formula supposes a threshold value

$$
c \approx 0.7 b \text {. }
$$

$b$ has been defined in (9) and is related to the dipole oscillator strength through (11). There is no evidence for a relation between $c$ and $b$ valid for all transitions and therefore we must reject (43a). For very high temperatures $(1 / \varepsilon \gg 1), P(\varepsilon) \approx(\sqrt{ } 3 / 2 \pi) E_{1}(\varepsilon)$ and it can be shown 
that (43a) and the high temperature limit of (34) become equal. This region is however of no physical interest because at such high temperatures the ion will be fully ionized to the next higher degree of ionization.

The parameters $A$ and $\chi$ in (43b) and (44b) have been given only for the transitions from the ground level to $n=2, n=3$ and to the continuum. For high $\varepsilon$ the factor containing $\chi$ is of little importance because it is of the order $0.2-0.9$. The rate coefficients do not differ very much from our results.

We cannot compare directly the formulae $44 a, 44 b$ and $44 c$ for ionization with our results, because we include also excitations to levels with $n \geq 8$, and therefore the lowtemperature behaviour of our ionization rate coefficients will not be as in formula 44 . The $n^{4}$ behaviour of $c^{\prime}$ in (44a) and (44c) is probably not far from the true $n$ dependence. Our empirical formulae (24) and (25) would indicate an $n^{13 / 3}$ relationship. Supposing the functional dependence of $c^{\prime}$ on $Z$ to be of the same form as in (44c), we adjusted the numerical constants to the measured ionization cross-sections for $Z=1$ and $Z=2$ and to the calculated cross-section for $Z=50$ (FITE and BRACKMANN ${ }^{(12)}$, DOLDER et al., ${ }^{(1)}$ BURGESS and RuDGE $\left.{ }^{(8)}\right)$. We suggest that $(44 \mathrm{c})$ be replaced by

$$
C_{\text {ion }}=3.0710^{-8}\left(2.42-\frac{1.72}{Z}+\frac{0.19}{Z^{2}}\right)(n / Z)^{4} \sqrt{ }(k T) \mathrm{e}^{-\varepsilon}
$$

The value of $A$ in (44b) as given by BEIGMANN $e t$ al. ${ }^{(1)}$ corresponds to $c^{\prime} \approx 4$ (the value predicted by the classical theory of Thomson, which is probably too high (cf. $44 \mathrm{c}^{\prime}$ ).

\section{REFERENCES}

1. I. Beigman, L. Vainshtein and A. Vinogradov. Astronom. J. (U.S.S.R.) 46, 985 (1969).

2. O. BeLY, J.I.L.A. Report 891966.

3. O. BeLY and F. BeLY, Sol. Phys. 2, 285 (1967).

4. O. Bely and M. Blaha, Sol. Phys. 3, 563 (1967).

5. H. Bethe, Ann. Phys. 5, 325 (1930).

6. A. Burgess, Mem. Soc. R. scient. Liege, $5^{\text {me }}$ serie 4, 299 (1961).

7. A. Burgess, H. van Regemorter and M. J. Seaton, unpublished. Cited by C. W. Allen, Mem. Soc. R. scient. Liege, $5^{\mathrm{me}}$ serie 4, 241 (1960).

8. A. Burgess and M. R. H. Rudge, Proc. R. Soc. A273, 372 (1963).

9. A. Burgess and M. J. Seaton, Mon. Not. R. Astr. Soc. 127, 355 (1964).

10. P. G. Burke, D. D. McVicar and K. Smith, Proc. Phys. Soc. 84, 749 (1964).

11. K. T. Dolder, M. F. A. Harrison and P. C. Thoneman, Proc. R. Soc. A264, 367 (1961).

12. W. L. Fite and R. T. Brackmann, Phys. Rev. 112, 1141 (1958).

13. M. InokutI, Argonne Natl. Lab. Rept. $6769,1963$.

14. M. InOKUTI and Y.-K. KIM, Abstracts of papers, VIth I.C.P.E.A.C., Cambridge, Mass., p. 51969.

15. M. Inokuti, Y.-K. Kim and R. L. Platzman, Phys. Rev. 164, 55 (1967).

16. A. JACOBS, JQSRT 11, 143 (1971).

17. L. D. Landau and E. M. Lifshrtz, Quantum Mechanics, Pergamon Press, 1959.

18. C. MAHN, I.P.P. (Garching) Rep. 3/52, (1967).

19. D. H. Menzel and C. L. Pekeris, Mon. Not. R. Astr. Soc. 96, 76 (1935).

20. W. F. Miller and R. L. Platzman, Proc. Phys. Soc. 70, 299 (1957).

21. B. L. MoiseiwitsCh and S. J. SMITH, Rev. Mod. Phys. 40, 238 (1968).

22. H. VAN Regemorter, Astrophys. J. 136, 906 (1962).

23. M. R. H. Rudge, Rev. Mod. Phys. 40, 564 (1968).

24. M. J. SeAton, Planet. Space Sci. 12, 55 (1964).

25. J. TUlLy, M.Sc. dissertation, London, 1960.

26. L. VRIEns and T. F. M. Bonsen, J. Phys. B, 1, 1123 (1968). 


\section{APPENDIX}

We shall indicate how the coefficients $a_{p}$ in (7) are computed. We start from the matrix element in (4) and choose the $z$-axis to be parallel to $\mathbf{k}$. We separate the matrix element in a finite sum of products of angular and radial parts by expanding the exponential $\mathrm{e}^{\text {ikrcoss }}$ in Legendre polynomials

$$
\left\langle n l m\left|\mathrm{e}^{i k r \cos s}\right| n^{\prime} l^{\prime} m^{\prime}\right\rangle=\sum_{\lambda} i^{\lambda}(2 \lambda+1)\left\langle l m\left|P_{\lambda}(\cos 9)\right|{ }^{\prime} m^{\prime}\right\rangle\left\langle n\left|j_{\lambda}(k r)\right| n^{\prime} l^{\prime}\right\rangle .
$$

The functions $j_{2}$ are spherical Bessel functions. The matrix elements of $P_{\lambda}$ can be calculated for instance by means of the formulae 29.3 and c. 14 of LANDAU and LIFSHITz. ${ }^{(17)}$ They are zero for $m \neq m^{\prime}$ and when the conditions

$$
\begin{aligned}
& \left|l-l^{\prime}\right| \leq \lambda \leq l+l^{\prime} \\
& l+l^{\prime}+\lambda \text { even }
\end{aligned}
$$

are not fulfilled.

$$
\begin{aligned}
\left\langle l m\left|P_{\lambda}(\cos \vartheta)\right| l^{\prime} m\right\rangle= & \sqrt{ }\left[\frac{(2 l+1)\left(2 l^{\prime}+1\right)(l-m) !\left(l^{\prime}+m\right) !}{(l+m) !\left(l^{\prime}-m\right) !}\right] \\
& \times(-1)^{\left(l-l^{\prime}+\lambda\right) / 2} \frac{\lambda !\left(l+l^{\prime}-\lambda\right) !\left[\left(l+l^{\prime}+\lambda\right) / 2\right] !}{\left(l+l^{\prime}+\lambda+1\right) !\left[\left(l^{\prime}-l+\lambda\right) / 2\right] !\left[\left(l+l^{\prime}-\lambda\right) / 2\right] !\left[\left(l-l^{\prime}+\lambda\right) / 2\right] !} \\
& \times \sum_{k}(-1)^{k} \frac{(l+m+k) !\left(l^{\prime}+\lambda-m-k\right) !}{(l-m-k) !\left(l^{\prime}-\lambda+m+k\right) !(\lambda-k) ! k !} .
\end{aligned}
$$

The summation over $k$ extends over all integer values of $k$ for which the factorials exist. The angular matrix elements may be expressed in terms of $3-j$-symbols :

$$
\left\langle l m\left|P_{\lambda}(\cos 9)\right| l^{\prime} m\right\rangle=(-1)^{m} \sqrt{ }\left[(2 l+1)\left(2 l^{\prime}+1\right)\right]\left(\begin{array}{lll}
l & l^{\prime} & \lambda \\
0 & 0 & 0
\end{array}\right)\left(\begin{array}{ccc}
l & l^{\prime} & \lambda \\
m & -m & 0
\end{array}\right) .
$$

The radial integral is

$$
\left\langle n l j_{\lambda}(k r) \mid n^{\prime} l^{\prime}\right\rangle=\int_{0}^{\infty} P_{n l}(Z, r) P_{n^{\prime} l^{\prime}}(Z, r) j_{\lambda}(k r) r^{2} \mathrm{~d} r .
$$

$P_{n}(Z, r)$ is the radial wave function of the hydrogen-like ion with nuclear charge $Z$. We change to the radial functions of the hydrogen atom through the substitutions $Z r \rightarrow r$ and $q=k / Z$ in (A4). The two radial wave functions consist each of an exponential and a polynomial in $r$ which can be multiplied giving

$$
P_{n l^{\prime}} P_{n^{\prime} l^{\prime}}=\frac{2^{l+l^{\prime}+2}(n-l-1) !\left(n^{\prime}-l^{\prime}-1\right) !}{n^{l+2} n^{l^{\prime}+2}} \mathrm{e}^{-a r} r^{l+l^{\prime}} \sum_{s=0}^{n+n^{\prime}-l-l^{\prime}-2} a_{s}\left(\frac{-2 r}{n^{\prime}}\right)^{s}
$$

with

$$
a_{s}=\sum_{p}\left(\begin{array}{l}
n^{\prime} \\
n
\end{array}\right)^{p}\left[(n-l-p-1) !\left(n^{\prime}-l^{\prime}+p-s-1\right) !(2 l+p+1) !\left(2 l^{\prime}+s-p+1\right) !(s-p) ! p !\right]^{-1}
$$

The sum over $p$ extends from the greater value among 0 and $s-n^{\prime}+l^{\prime}+1$ to the smaller value among $s$ and $n-l-1$.

The integration over $r$ in (A4) can now be carried out by means of the formula

$$
\begin{aligned}
\int_{0}^{\infty} \mathrm{e}^{-a r} r^{p} j_{\lambda}(q r) \mathrm{d} r= & \alpha^{-p-1} \int_{0}^{\infty} \mathrm{e}^{-r} r^{p} j_{\lambda}(r \sqrt{ } x) \mathrm{d} r=\alpha^{-p-1}(2 \sqrt{ } x)^{\lambda} \sum_{t}(x+1)^{(-t-p-\lambda-1) / 2}(-1)^{(t-p+\lambda+1) / 2} \\
& \times 2^{t} \frac{(p-\lambda-1) ![(\lambda+p+1+t) / 2] !}{t ![(p-\lambda-1-t) / 2]}
\end{aligned}
$$

The sum over $t$ extends over those values of $t$ for which

$$
0 \leq t \leq p-\lambda-1
$$

and

$$
t+p+\lambda+1 \text { even. }
$$

A few simple algebraical operations as taking out the common denominator $(x+1)^{n+n^{\prime}}$, ordering in powers of $x$ and squaring finally lead to (7). 